\title{
Caregiver Response to an Online Dementia and Caregiver Wellness Education Platform
}

\author{
Katie Rodriguez ${ }^{\mathrm{a}, \mathrm{b}}$, Madison Fugard ${ }^{\mathrm{c}}$, Shawna Aminib,d ${ }^{\mathrm{b}}$, Glenn Smith ${ }^{\mathrm{a}}$, Deann Marasco ${ }^{\mathrm{e}}$, \\ Julie Shatzer ${ }^{\mathrm{e}}$, Michelle Guerrero ${ }^{\mathrm{c}}$, Cynthia Garvan ${ }^{\mathrm{b}, \mathrm{d}}$, Jonathan Davis ${ }^{\mathrm{c}, *}$ and Catherine Price ${ }^{\mathrm{a}, \mathrm{b}, \mathrm{d}, *}$ \\ ${ }^{a}$ Clinical and Health Psychology, University of Florida, Gainesville, FL, USA \\ ${ }^{\mathrm{b}}$ Perioperative Cognitive Anesthesia Network for Alzheimer's Disease and Related Dementias, \\ University of Florida, Gainesville, FL, USA \\ ${ }^{\mathrm{c}}$ Trualta Incorporated, Ottawa, Ontario, Canada \\ ${ }^{\mathrm{d}}$ Anesthesiology, University of Florida, Gainesville, FL, USA \\ 'Alzheimer's Association, FL, USA
}

Accepted 19 April 2021

Pre-press 16 May 2021

\begin{abstract}
.
Background: Web-based educational interventions are emerging as a potential solution to improve caregiver dementia knowledge and overall well-being.

Objective: To assess the feasibility of delivering a web-based intervention for dementia caregivers by examining: 1) engagement with the online platform, 2) skill implementation, and 3) changes on outcome metrics over the 30-day study period.

Methods: Enrolled participants were onboarded by a trained research coordinator and provided 24/7 access to the platform over 30 days. At study onset and completion, caregivers completed assessments of care recipient dementia severity and neuropsychiatric symptoms along with instruments which measured dementia knowledge, caregiver burden, and carer experience.

Results: Of 84 referrals, 60 caregivers met study inclusion criteria and 55 completed pre and post study measures. Caregivers completed an average of 8 hours of learning over the 30-day web-based intervention, with $84.4 \%$ of participants reporting using at least one skill they learned from the online platform. Eighty-nine percent of participants reported high satisfaction with the web-based educational intervention. There were small effect sizes for decreases in NPIQ neuropsychiatric symptom severity and caregiver distress scores from pre- to post-intervention. Small effect sizes were observed for changes in caregiver burden from pre- to post-intervention among caregivers who perceived their care recipient as having high global deterioration. Conclusion: Findings show online educational programs are feasible for informal family caregivers of dementia and have perceived value. Future studies should address caregiver response to online education in less severe versus more severe care recipients, and explore the value of caregiver online platforms in diverse caregiver samples.
\end{abstract}

Keywords: Alzheimer's disease, caregiver burden, dementia knowledge, feasibility studies, internet-based intervention, personal satisfaction

\footnotetext{
*Correspondence to: Catherine Price, $\mathrm{PhD}$, Associate Professor, Clinical and Health Psychology, University of Florida, Gainesville, FL, 32610, USA. Tel.: +1 352273 5929; E-mail: cep23@phhp.ufl.edu. and Jonathan Davis, CEO, Trualta Inc., Ottawa, Ontario, Canada. E-mail: jonathan@trualta.com.
}

As the population continues to age in the United States and elsewhere, dementia has become a significant social, economic, and public health concern. Family members often take on the role of caring for a person with dementia at home [1]. Individuals with dementia often require assistance with a variety of daily activities. This care is typically provided by 
informal caregivers. Informal dementia caregivers are often family members or friends that provide unpaid care to someone with dementia. In fact, $83 \%$ of older adults with dementia receive informal assistance from family members, friends, or other unpaid caregivers [2]. In addition to instrumental and basic activities of daily living, family members are often tasked with managing the behavioral symptoms of dementia, such as agitation, rejection/refusal of care, and apathy [2]. These behavioral and psychological symptoms of dementia (BPSD) are typically common throughout the disease trajectory and are especially challenging for both the person with dementia and their caregiver [3].

Given the primary role family members play in providing care, the development of interventions to support the family caregivers are critical in the management of care for persons with dementia. Currently, there is no widely available solution that meets informal caregiver education needs to allow family caregivers to access and actively use the information that may be most useful to them at the time that they need it $[4,5]$. Even in mild stages of cognitive impairment, educational interventions appear to be among the most important nonpharmacological interventions, not only to reduce caregiver anxiety and burden, but also to improve patient mood and quality of life [6, 7]. In a review of empirical studies addressing nonpharmacological interventions to decrease BPSD, Caspar and colleagues identified three mechanisms as necessary for sustained effective outcomes: the caring environment, care skill development and maintenance, and individualization of care [5]. The authors concluded nonpharmacological interventions should include consideration of both the physical and the social environment, as well as ongoing education and support for care providers. If family caregiver information needs are not met, then family members cannot provide effective care for a person with dementia [8]. Werner et al. also reported caregivers need information to be provided: 1) in a timely manner, 2) tailored to individual needs, and 3 ) in a usable fashion [9]. Interventions providing education and practical information have been shown to improve caregiver knowledge, decrease stress/workload, and empower caregivers within the healthcare system [10-12].

In recent decades, web-based educational interventions have emerged as a potential solution to meet the unique information needs of family caregivers [11]. Online interventions are advantageous in their opportunity for increased access, scalability, and cost-efficiency and have the potential to reach more remote/rural populations who struggle to access faceto-face services $[13,14]$. Web-based education and support programs have also shown effectiveness in improving caregiver knowledge and mental health, and in reducing burden among caregivers of people with dementia [12]. While most online interventions demonstrate some efficacy in adapting existing education programs into structured sessions/videos or via virtual facilitation of caregiver support groups, several studies have identified the need for more personalized education, with content specific to caregivers' unique care situation and preferences $[9,10$, $15,16]$.

Implementation of web-based interventions for dementia caregivers presents its own set of challenges, however. Changes in cognitive, motor, and perceptual function coupled with the fast-paced evolution of technology is a considerable barrier for caregivers among the aging population [17]. Given the age range of informal caregivers, online interventions are responsible for accommodating the technological needs of individuals across the lifespan. Additionally, current literature on web-based interventions for dementia caregivers focuses disproportionally on the trial phase rather than the intervention making it increasingly difficult to understand the trajectory of online interventions [11]. Thus, there is a particular need for a centralized information hub that provides caregiver education materials that fulfill specific self-defined needs and can be implemented in practice.

The research team of the current study designed a quasi-experimental study to determine the feasibility and perceived value of delivering a remote, webbased caregiver intervention with different learning modules. There were three study aims. First, to examine caregivers' engagement with the web-based educational platform during a period of 30 days from time of enrollment. Second, to examine if caregivers reported using the skills learned from the online platform and were satisfied with this platform. Third, to explore if caregivers reported a change in burden, carer experience, distress, and perceived severity of neuropsychiatric symptoms based on the dementia severity of the care recipient.

\section{METHODS}

The current study was designed to assess the feasibility of delivering a web-based intervention for 
dementia caregivers. Feasibility studies are used to assess the parameters under which a proposed study will work. To do this, the University of Florida study team (consisting of clinical neuropsychologists and research coordinators) partnered with the Alzheimer's Association, Florida, and Trualta ${ }^{\mathrm{TM}}$ to examine: 1) engagement with the online platform, 2) skill implementation, and 3) changes on outcome metrics over the 30-day study period. Caregivers were recruited and enrolled during the COVID-19 pandemic. Study procedures were approved by the University of Florida Institutional Review Board. Eligible participants completed econsents before completing screening criteria and study enrollment. Methods were in accordance with the Declaration of Helsinki.

\section{Participants}

Program managers affiliated with the Alzheimer's Association, Florida, provided IRB approved study information to informal dementia caregivers throughout the months of May through July 2020. Interested caregivers were referred to UF study personnel who provided additional study information, reviewed inclusion criteria, reviewed consent forms, and enrolled eligible participants. In order to participate in the study, participants were required to meet the following inclusion criteria: age 18 and older, fluent in written and verbal English, listed within a non-profit registry for dementia caregivers, serving as an active informal caregiver for an individual with a concern of cognitive impairment (heretofore called 'care recipient'), access to an electronic device for education purposes, willing to complete baseline and follow-up assessments, and had to demonstrate computer proficiency by answering yes to each item of a modified version of a computer proficiency scale (CPQ-12) [18].

\section{Procedures}

Trained staff members completed remote onboarding with caregivers. The onboarding process is an orientation to the learning platform and was completed using video conferencing and screensharing technologies to demonstrate to caregivers how to select a display name, complete profile registration, and navigate different types of modules available on the platform. To supplement the online intervention, participants were also mailed print material regarding the web-based educational platform. The print material included three print booklets that serve as examples of the 64 training modules included in the online platform: Aging \& Brain Health, Safety and Injury Prevention, and Personal Care. Time stamped system generated data were acquired based on logins, module views (page loads), completed modules with quizzes, and user comments. To confirm knowledge of the onboarding process, trained staff completed a follow-up check-in at 72 hours after onboarding, 14 days after onboarding to assess changes in living situation and troubleshooting problems, and again at 30 days to complete outcome metrics. Caregivers were given 24/7 access to the platform over the 30-day study period and were able to log in on a computer, laptop, tablet, or smartphone.

Prior to engaging with the web-based educational platform, participants completed the study measures which included assessments of caregiver burden, carer experience, caregiver distress, and caregiver perceived severity of neuropsychiatric symptoms of the care recipient. After 30 days of study participation, participants completed the skill implementation questionnaire, a questionnaire assessing their satisfaction with the web-based educational platform, and the study measures administered at baseline. These questionnaires were completed online using REDCap database [19].

\section{Materials and measures}

\section{Web-based educational platform}

Caregiver education was provided through Trua$1 \mathrm{ta}^{\mathrm{TM}}$ - a personalized web-based platform that was developed to provide informal caregivers with access to a unique learning journey through a comprehensive library of multi-modal education modules. Modules address a variety of topics identified as important by caregivers in previous literature including behavioral symptom management, skills training for basic activity assistance, safety management, and caregiver wellness [10]. Modules provided to the caregivers were developed by experts in the field of nursing, care management, neuropsychology, occupational therapy, and social work.

Caregivers were provided with specific module recommendations relevant to his or her care situation based on: 1) topics selected during initial registration using a system generated program and 2) caregiver feedback given to the research coordinator throughout the 30 days of study enrollment. Supplementary Table 1 lists available Trualta $^{\mathrm{TM}}$ modules. 


\section{Assessment of caregiver engagement and learning}

Time-stamped activity logs. Within the electronic portal, deidentified information was captured regarding activity involving module views, quizzes, comments, and badges for skill completion. Each activity was assigned a "seat time" indicating the total time required to complete that activity. Outcome variable: duration of activities performed in the portal.

Skill implementation. At the end of the 30-day study, caregivers were asked, "Have you used what you learned in Trualta $^{\mathrm{TM}}$ to help with: 1) Personal care activities (like helping with bathing, grooming, or mealtime); 2) Manage behaviors that happen with memory loss or dementia (like changes in aggression, or mood); 3) Manage your own care and wellness (like self-care, and reducing your stress); 4) Promote safety at home (like preventing falls, and moving safely); and 5) Understand local resources, like accessing services in my community such as support groups". Answers were one of four options: "not at all", "a few times a week", "everyday", "not applicable to the person I care for".

\section{Questionnaires to understand caregiver characteristics}

Caregivers completed the following measures assessing caregiver burden and carer experience at baseline and post-intervention.

The Dementia Knowledge Assessment Tool Version Two (DKA-T2). The DKA-T2 is a 21 item assessment of foundational knowledge of the dementia trajectory and care in formal and informal caregivers [20]. Correct scores are totaled to provide an indication of the caregivers' overall baseline dementia knowledge.

The Zarit Caregiver Burden Interview Short Form (ZBI-12). The ZBI-I2 is a 12-item questionnaire that assesses perceived caregiver burden associated with caregiving for a dependent patient [21]. Items target psychological, physical, economical, and communication problems that cause distress and burden for the caregiver. Items are scored on a five-point Likert scale from 0 (never) to 4 (almost always). Total burden mean score is calculated by summing all responses. Higher mean scores represent greater perceived caregiver burden with suggested cut-offs of 0-10 (no to mild burden), 10-20 (mild to moderate burden), > 20 (high burden) (Supplementary Table 2).

The Carer Experience Scale (CES). The CES is a preference-based questionnaire designed to assess care-related quality of life and consists of six conceptual attributes: activities outside caring, social support, institutional support, fulfillment from caring, control over caring, and getting along with the care recipient [22]. Each attribute has three response levels, representing three levels of frequency ("rarely", "sometimes", "mostly") or amount ("a little", "some", "a lot"). An index value score is calculated by summing the utility weights for each level of the six attributes, with higher scores reflecting better caring states. CES response values are reported in Supplementary Table 3.

\section{Recipient characteristics and disease severity}

Caregivers completed the following measures assessing recipient severity characteristics.

Global Deterioration Scale (GDS). The GDS assesses the severity of dementia and its stages of cognitive decline [23]. The GDS divides the course of dementia into seven stages, ranging from no cognitive decline (stage 1) to very severe cognitive decline (stage 7). Each stage corresponds to clinical phases of dementia, from normal (stage 1) to late dementia (stage 7), and are accompanied by a short description of characteristics. [24, 25].

Neuropsychiatric Inventory Questionnaire (NP $I Q$ ). The NPIQ is a retrospective instrument used to measure caregivers' perceptions of their care recipient's severity of neuropsychiatric symptoms and caregivers' distress related to these neuropsychiatric symptoms over the past month [26]. The 12 neuropsychiatric symptoms include: delusions, hallucinations, agitation/aggression, dysphoria/depression, anxiety, euphoria/elation, apathy/indifference, disinhibition, irritability/lability, aberrant motor behaviors, night-time behavioral disturbances and appetite/ eating disturbances. The questionnaire is filled out by the care recipient's primary caregiver. Responses assess the caregiver's perception of the presence, frequency, severity, and distress of neuropsychiatric symptoms of the care recipient. Caregivers are first asked to indicate the presence of the neuropsychiatric symptoms (yes/no). Caregivers who report the presence of a symptom are then asked to rate the severity of the symptom and caregiver distress associated with the symptom. Severity of neuropsychiatric symptoms is rated on a three-point scale ("mild", "moderate", and "severe") and caregiver distress is rated on a fivepoint scale ("no distress:", "minimal distress", "mild distress", "moderate distress", "severe distress" and "extreme distress"). Items assessing severity and distress were summed to create a total domain score, with higher values representing greater severity and distress, respectively (Supplementary Table 4). 


\section{Satisfaction exit questionnaire}

Caregivers were asked if they would recommend an online education platform like the one used in this study to another dementia caregiver, with the rating on a 10-point Likert scale 1 (not likely) to 10 (very likely).

\section{Statistical approach}

Analyses were conducted using all valid primary outcome data. Missing values $(<1 \%)$ on secondary outcomes (caregiver burden, carer experience, perceived severity of neuropsychiatric symptoms, and caregiver distress) were replaced using participants' mean score. Analyses were conducted with and without missing values and produced the same pattern of results. The displayed results reflect analyses conducted without missing values. Analyses for caregiver burden (Zarit), and carer experience (CES) were conducted with all 55 participants. Analyses for perceived severity of neuropsychiatric symptoms (NPIQ) and caregiver distress (NPIQ) were conducted on 54 participants because one participant reported that their care recipient did not have any neuropsychiatric symptom, and therefore did not report

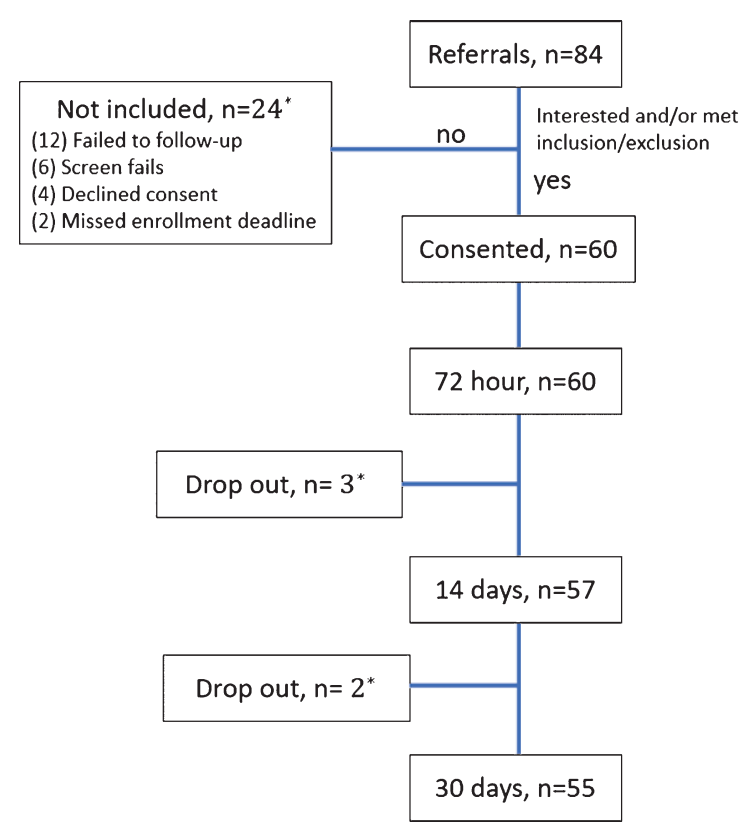

Fig. 1. Participant recruitment and inclusion flow. * Not included (12 failed to follow-up for more information; 6 did not meet inclusion criteria, 4 declined consent, and 2 missed the enrollment deadline); Post 72 hour drop out (2 lost to follow-up; 1 asked to withdraw due to time constraints); Post 14 day drop out (1 lost to follow-up; 1 asked to withdraw due to time constraints). distress associated with the presence of neuropsychiatric symptoms.

Descriptive statistics were calculated for the primary outcomes of engagement with web-based platform, skill implementation, and caregiver satisfaction with web-based platform. Analyses for the outcomes of changes in caregiver burden, carer experience, perceived severity of neuropsychiatric symptoms, and caregiver distress were conducted for the entire sample as well as separately for caregivers with a loved one with less disease severity (answered 2 or 3 on the GDS) and caregivers with a more severe recipients (answered 4 to 7 on the GDS). Paired sample $t$-tests were conducted to examine differences in caregiver burden, carer experience, perceived severity of neuropsychiatric symptoms, and caregiver distress from pre- to post-online education for the entire sample and for each group. Statistical analyses were conducted in SPSS 25. The false discovery rate of $p<0.05$ for multiple comparisons and Cohen's $d$ effect size were used to assess the significant effects. Effect sizes of $0.20,0.50$, and 0.80 were considered small, medium, and large, respectively.

\section{RESULTS}

\section{Participants}

Of the possible caregivers throughout the dementia registry, 84 were referred to the study team. Sixty out of 84 caregivers moved forward with study enrollment. Twelve caregivers failed to follow-up with the study team after initial contact was made, six caregivers did not meet study inclusion criteria (i.e., screen fails), four caregivers declined to participate, and two caregivers missed the enrollment deadline. Fifty-five participants completed both pre- and poststudy measures (see Fig. 1). Descriptive statistics are displayed in Table 1. Caregivers were mostly women $(78 \%)$, spouses $(49 \%)$ or children $(32 \%)$, and white $(75 \%)$. The majority of caregivers lived with the person they were caring for at the time of the study (72\%). The age range of caregivers was 37 to 81 years and education was dominantly high school or greater. Caregivers most often reported the care recipient as having "moderately severe cognitive decline" (42\%), followed by "moderate cognitive decline" (18\%), "severe cognitive decline" (16\%), "mild cognitive decline" (15\%), "very mild cognitive decline" (7\%), and "very severe cognitive decline" $(2 \%)$. According to the NPIQ, caregivers listed memory and thinking problems as mild (26\%), moderate 
Table 1

Demographics of caregivers

\begin{tabular}{|c|c|}
\hline Caregiver Characteristic & $\begin{array}{c}\text { Mean (SD) or } \%(n) \\
n=55\end{array}$ \\
\hline$\overline{\text { Age }}$ & $64.96(10.9)$ \\
\hline Education $(y)$ & $16.24(2.32)$ \\
\hline \multicolumn{2}{|l|}{ Sex } \\
\hline Male & $22 \%(13)$ \\
\hline Female & $78 \%(42)$ \\
\hline \multicolumn{2}{|l|}{ Race } \\
\hline White & $76 \%(42)$ \\
\hline Black & $9 \%(5)$ \\
\hline Hispanic & $10 \%(6)$ \\
\hline Other & $3 \%(2)$ \\
\hline \multicolumn{2}{|l|}{ Relationship to the care recipient } \\
\hline Spouse/partner/companion & $50 \%(28)$ \\
\hline Child & $30 \%(17)$ \\
\hline Sibling & $1 \%(1)$ \\
\hline Other relative & $16 \%(9)$ \\
\hline $\begin{array}{l}\text { Time spent living with } \\
\text { the care recipient }\end{array}$ & $70 \%(38)$ \\
\hline \multicolumn{2}{|l|}{$\begin{array}{l}\text { Perceived dementia severity } \\
\text { of care recipient }\end{array}$} \\
\hline Moderately severe cognitive decline & $42 \%(23)$ \\
\hline Moderate cognitive decline & $18 \%(10)$ \\
\hline Severe cognitive decline & $16 \%(9)$ \\
\hline Mild cognitive decline & $15 \%(8)$ \\
\hline Very mild cognitive decline & $7 \%(4)$ \\
\hline Very severe cognitive decline & $2 \%(1)$ \\
\hline \multicolumn{2}{|l|}{ Employment status } \\
\hline Employed part-time & $16 \%(9)$ \\
\hline Employed full time & $12 \%(7)$ \\
\hline Unemployed, looking for work & $3 \%(2)$ \\
\hline Unemployed, not looking for work & $1 \%(1)$ \\
\hline Retired & $65 \%(36)$ \\
\hline
\end{tabular}

(50\%), or severe (24\%). On the NPIQ, more than half of the participants reported observing problematic behaviors (agitation/aggression, anxiety, apathy, irritability/lability, nighttime behaviors, appetite/eating) with approximately a third of the sample reporting disinhibition, delusions, depression, disinhibition. Fewer than $10 \%$ reported observing hallucinations and elation/euphoria.

\section{Online platform engagement, skill implementation, and satisfaction}

\section{Engagement}

Caregivers, on average, completed 31 educational modules which is equivalent to 8 hours of learning on the portal. Older caregivers ( $\geq 60$ years) averaged 34 modules and 8.13 hours per user during the 30 -day study period while those less than 60 years averaged 28 modules and 7.28 hours per user. Level of engagement did not differ across patient disease severity $(U=252.50, p=0.911)$ or caregiver-patient relationship $(F=2.05, p=0.118)$. Furthermore, level of engagement was not correlated with years of education ( $r=-0.03, p=0.845)$ or caregiver income $(r=0.07, p=0.632)$. Table 2 displays time spent on each module.

Skill implementation. Ten individuals had missing questionnaire items or did not complete the questionnaire, and reported statistics do not include these individuals. Analyses were conducted to determine whether participants with missing data on the skill implementation questionnaire differed from those with complete data. Multiple comparisons were corrected using a false discovery rate. Results showed the two groups (i.e., those with missing data vs. those without missing data) were not found to differ on: caregiver age $(t=0.75, p=0.454)$, caregiver sex $\left(\chi^{2}=0.90, p=0.765\right)$, caregiver income $(U=206.00$, $p=0.753)$, caregiver education $(t=0.05, p=0.957)$, caregiver race $(U=156.00, p=0.043)$, caregiver relationship $(U=160.00, p=0.123)$, and patient disease severity $\left(\chi^{2}=0.48, p=0.489\right)$. Of those who completed the questionnaire $(n=45), 10$ reported using one module skill, four indicated using two skills, nine indicated three skills, nine indicated using four skills, and six participants indicated five skills, i.e., 84.4\% (38/45) participants used at least one of the five module-based training skills. Four participants reported that they did not use any of the skills, while three participants reported a combination of not using the skill and the skill not being applicable to their care recipient. Table 2 displays the modules by reported use. The module addressing behavioral management behaviors with memory loss or dementia (e.g., changes in aggression or mood) reported the highest frequency $(60 \% ; 27 / 45)$, followed by the module addressing caregiver wellness (55.6\%; $25 / 45)$, personal care activities $(46.7 \% ; 21 / 45)$, safety at home $(44.44 \% ; 20 / 45)$, and local resources $(42.2 \%$; 19/45).

Satisfaction. The majority of caregivers reported they would recommend an online educational platform like Trualta ${ }^{\mathrm{TM}}$ to another dementia caregiver in a similar care situation with $60 \%(n=33)$ rating it as a $10 / 10$ on a 10 -point Likert scale, $16 \%(n=9)$ rating it as a $9 / 10,13 \%(n=7)$ rating it as an $8 / 10,7 \%(n=4)$ rating it as a $7 / 10$, and $4 \%(n=2)$ rating it as a $6 / 10$.

\section{Changes in outcome metrics}

\section{Entire sample}

There were no significant changes from pre- to post-intervention in perceived severity of neuropsychiatric symptoms (NPIQ), $t(53)=2.439, p=0.018$, Cohen's $d=0.332$ (95\% CI: 0.06, 0.61), caregiver 

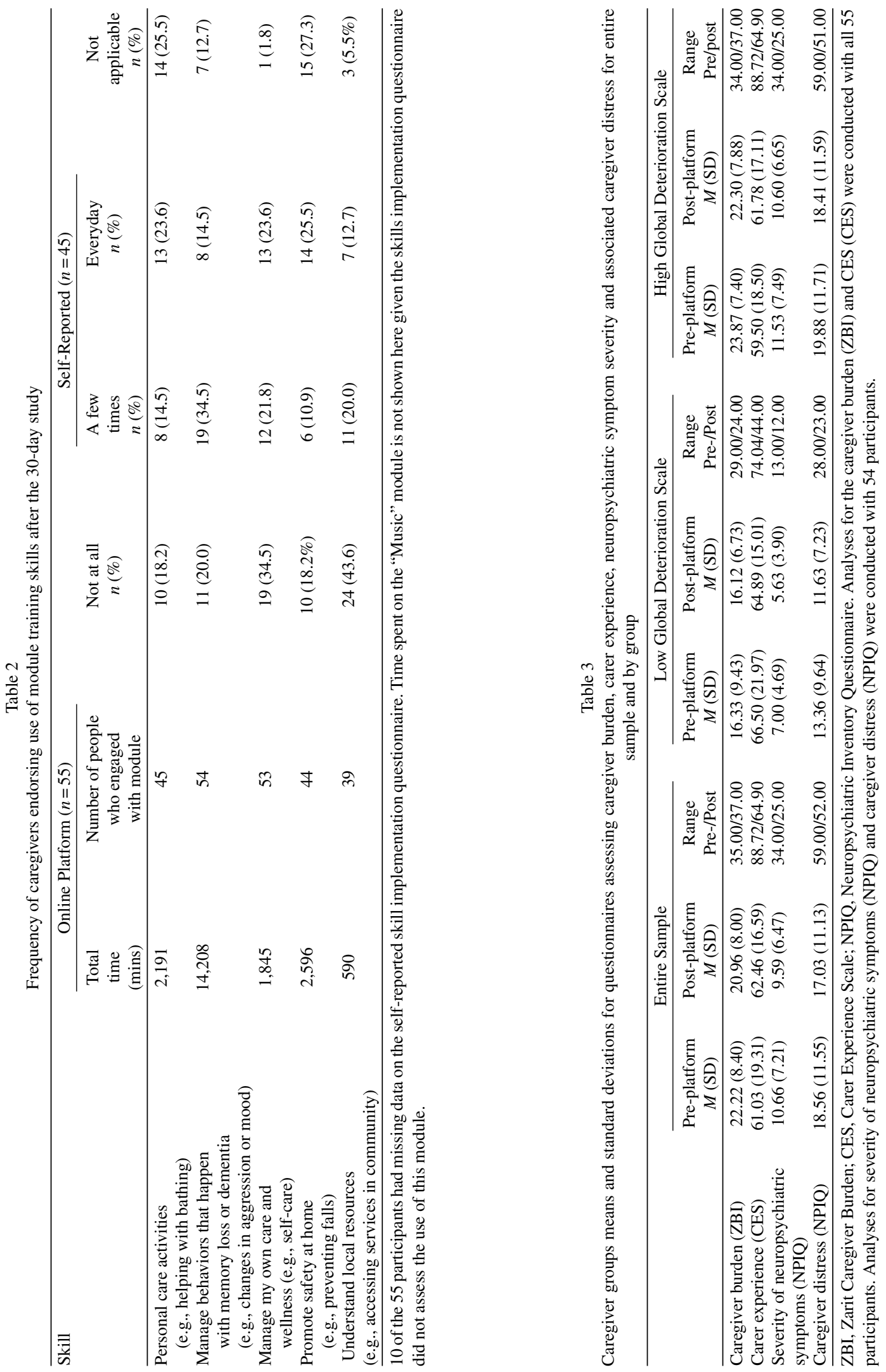
distress (NPIQ), $t(53)=2.129, p=0.038$, Cohen's $d=0.290$ (95\% CI: 0.02, 0.56), caregiver burden (Zarit), $t(54)=1.868, p=0.067$, Cohen's $d=0.252$ (95\% CI: $-0.02,0.52$ ), or carer experience (CES), $t(54)=-0.698, p=0.488$, Cohen's $d=-0.094(95 \%$ CI: $-0.36,0.17$ ).

By group. For the low GDS group, paired $t$-tests showed no significant change from pre- to postintervention in perceived severity of neuropsychiatric symptoms (NPIQ), $t(10)=2.193, p=0.053$, Cohen's $d=0.661$ (95\% CI: $-0.01,1.30)$, caregiver distress (NPIQ), $t(10)=1.350, p=0.207$, Cohen's $d=0.407$ (95\% CI: $-0.22,1.01$ ), caregiver burden (Zarit), $t(11)=0.101, p=0.921$, Cohen's $d=0.029(95 \% \mathrm{CI}$ : $-0.54,0.59$ ), or carer experience (CES), $t(11)=0.656$, $p=0.656$, Cohen's $d=0.132$ (95\% CI: $-0.44,0.70$ ). For the high GDS group, Paired $t$-tests showed no significant changes from pre- to post-intervention in perceived severity of neuropsychiatric symptoms (NPIQ), $t(42)=1.854, p=0.071$, Cohen's $d=0.283$ (95\% CI: $-0.02,0.59$ ), or caregiver distress (NPIQ), $t(42)=1.744, p=0.088$, Cohen's $d=0.266$ (95\% CI: $-0.04,0.57$ ), caregiver burden (Zarit), $t(42)=2.131$, $p=0.039$, Cohen's $d=0.325$ (95\% CI: 0.02, 0.63) or carer experience (CES), $t(42)=-0.935, p=0.355$, Cohen's $d=-0.143$ (95\% CI: $-0.44,0.16$ ).

\section{DISCUSSION}

The overall purpose of the current study was to determine the feasibility of delivering a remote, webbased caregiver intervention. Results showed that caregivers were willing to use the web-based platform and did so for an average eight hours over the course of the intervention. Results also showed that caregivers reported applying the skills provided through the educational modules. An exploration into caregiver type based on dementia severity (GDS) suggests clinically significant trends for decreases in neuropsychiatric severity and caregiver distress after 30 days on the on-line educational platform.

The caregivers within our cohort were mostly white $(75 \%)$ and female $(78 \%)$. Consistent with the literature, these individuals were family members and more often spouses (49\%) [27]. Drop-out was minimal (91.6\% retention) over the period of 30 days. Despite being busy (70\% of their time reported as spent caregiving) and knowledgeable of dementia $(67.10 \%$ of questions were answered correctly [14.09/21] on DKA T2), caregivers in the current study were willing to engage throughout the 30- day period. On average, caregivers spent a total of eight hours using the online platform, with older caregivers ( $\geq 60$ years) spending more time on the platform (8.13 hours) than younger adults $(<60$ years; 7.28 hours). Although this study demonstrated engagement for one month with an online portal to educational training, it is unknown how caregivers will use or benefit from the personalized online caregiver portal for longer than 30 days.

Of the participants who completed the 30-day skill assessment questionnaire, $84.4 \%$ reported using skills that were acquired through the on-line platform. The highest percent of use was for skills pertaining to behavioral management $(60 \%)$, which is consistent with other research showing caregiver need for how to manage neuropsychiatric symptoms [3]. Caregivers $(55.6 \%)$ also reported allocating more time for self-care, featuring a particularly positive result of the intervention considering limited self-care can often lead to situations of crisis for dementia caregivers, causing them to rely more on emergency or home care services [28]. In addition, nearly half of the caregiver population used skills they learned to address personal care activities, promote safety, and access local resources. This highlights the potential for online interventions to help informal caregivers' access valuable techniques they can apply in their daily life. Participants with missing data on the skill implementation questionnaire did not differ from those with complete data on caregiver age, sex, income, education, race, caregiver relationship, and patient disease severity.

Analyses conducted with the entire sample and subsamples showed no significant score changes from pre- to post-online training. For the entire sample, however, there were small effect sizes for decreases in NPIQ neuropsychiatric symptom severity and caregiver distress scores from pre- to postintervention. This finding provides preliminary evidence that a personalized, educational web-based training program may decrease perceptions of recipient neuropsychiatric severity and caregiver distress. When post hoc analyses were conducted separately based on perceived caregiver severity of recipient (i.e., low versus high global deterioration), a small effect size suggested caregivers who perceived his/her recipient as having high global deterioration had a lower post-training burden score relative to the pre-training score. Several studies have noted that caregiver burden increases with increasing severity of disease [29, 30]. Educational online training programs may therefore most assist with burden 
reduction for caregivers reporting higher burden and perceiving the recipient as more impaired. While we acknowledge that additional investigations with larger and more diverse samples are needed to confirm the clinical significance of our study findings, we interpret the general pre-post comparisons as evidence of potential value for personalized educational platforms.

We recognize study limitations. First, the caregiver participants were largely Caucasian, older, and spouses of the recipient. This is a limitation, as the research is not representative of the caregiver cohort of the United States [31]. Experiences of caregiving differ based on ethnicity and relationship to the care recipient, and it is not known how these factors may impact engagement with web-based interventions [32-34]. Second, online engagement was assessed for a limited period of 30 days, which is a limitation relative to programs ranging from 2 to 3 months, on average [35]. Based on the dementia knowledge scale completed prior to on-line boarding, the caregivers were also highly knowledgeable about dementia which may have biased study findings. Caregivers may have engaged with the platform more frequently knowing it was an expectation of the study, and may have over reported skill use and symptom improvement (e.g., Rosenthal effect) [36]. Third, caregivers' level of distress may be correlated with their perceptions of neuropsychiatric symptom severity, and therefore low/high perceived distressed may be result in less/more reporting of symptoms. Finally, the study was conducted during the early months of the COVID19 pandemic, and it is unknown if the environmental situation involving isolation may have biased response to the online portal.

Despite these considerations, this investigation demonstrates feasibility for caregiver engagement in a personalized web-based training platform and shows potential value for improved dementia care skill use and possibly reduced burden even for more caregivers of more severely compromised care recipients. The study also suggests caregivers of less severe versus more severe recipients may respond differently to online platform training. These findings highlight the need for research on caregiver type and also the value for tailored caregiver training options. Finally, the study received excellent recruitment (60 out of 84 recruited; $71.4 \%$ recruited) with minimal dropout. The satisfaction questionnaire indicates caregivers were satisfied with the platform and would recommend this or a similar platform to other caregivers in similar situations. Given the impact of dementia on social, economic and public health care, study findings highlight the need for caregiver online educational platforms for support and skill training.

\section{ACKNOWLEDGMENTS}

We sincerely thank the care managers with the Florida Alzheimer's Association. Without their engagement and interest in this project, the study would not have been possible during the stressful COVID19 pandemic time period. We also sincerely appreciate every caregiver and patient who considered the study and participated in the investigation.

Funding provided by Canadian Centre for Aging and Brain Health Innovation (CABHI). CABHI provides funding and support to innovators for the development, testing, and dissemination of new ideas and technologies that address unmet brain health and seniors' care needs.

\section{CONFLICT OF INTEREST}

This project was conducted as part of a grant funding opportunity designed to partner innovators devoted to unmet brain health and senior care needs with academic affiliated researchers. The following authors are associated with Trualta ${ }^{\mathrm{TM}}:$ Jonathan Davis, Trualta ${ }^{\mathrm{TM}}$ Founder and CEO; Madison Fugard, MScOT; Michelle Guerrero, Ph.D.

Care was taken to integrate the Trualta ${ }^{\mathrm{TM}}$ business with unaffiliated researchers without conflict of interest. Biostatistical analyses were conducted separately and then jointly between researchers and biostatisticians from both parties.

\section{SUPPLEMENTARY MATERIAL}

The supplementary material is available in the electronic version of this article: https://dx.doi.org/ 10.3233/ADR-200292.

\section{REFERENCES}

[1] Callahan CM, Tu W, Unroe KT, LaMantia MA, Stump TE, Clark DO (2015) Transitions in care in a nationally representative sample of older Americans with dementia. $J \mathrm{Am}$ Geriatr Soc 63, 1495-1502.

[2] (2020) 2020 Alzheimer's disease facts and figures. Alzheimers Dement 16, 391-460.

[3] Mavounza C, Ouellet MC, Hudon C (2020) Caregivers' emotional distress due to neuropsychiatric symptoms of persons with amnestic mild cognitive impairment or Alzheimer's disease. Aging Ment Health 24, 423-430. 
[4] Gitlin LN, Kales HC, Lyketsos CG (2012) Nonpharmacologic management of behavioral symptoms in dementia. JAMA 308, 2020-2029.

[5] Caspar S, Davis ED, Douziech A, Scott DR (2018) Nonpharmacological management of behavioral and psychological symptoms of dementia: What works, in what circumstances, and why? Innov Aging 2, igy001.

[6] Chandler MJ, Locke DE, Crook JE, Fields JA, Ball CT, Phatak VS, Dean PM, Morris M, Smith GE (2019) Comparative effectiveness of behavioral interventions on quality of life for older adults with mild cognitive impairment: A randomized clinical trial. JAMA Netw Open 2, e193016.

[7] Amofa PA, Locke DEC, Chandler M, Crook JE, Ball CT, Phatak V, Smith GE (2021) Comparative effectiveness of behavioral interventions to prevent or delay dementia: Oneyear partner outcomes. J Prev Alzheimers Dis 8, 33-40.

[8] Steiner V, Pierce LL, Salvador D (2016) Information needs of family caregivers of people with dementia. Rehabil Nurs 41, 162-169.

[9] Werner NE, Stanislawski B, Marx KA, Watkins DC, Kobayashi M, Kales H, Gitlin LN (2017) Getting what they need when they need it. Identifying barriers to information needs of family caregivers to manage dementia-related behavioral symptoms. Appl Clin Inform 8, 191-205.

[10] Whitlatch CJ, Orsulic-Jeras S (2018) Meeting the informational, educational, and psychosocial support needs of persons living with dementia and their family caregivers. Gerontologist 58, S58-S73.

[11] Christie HL, Bartels SL, Boots LMM, Tange HJ, Verhey FJJ, de Vugt ME (2018) A systematic review on the implementation of eHealth interventions for informal caregivers of people with dementia. Internet Interv 13, 51-59.

[12] Egan KJ, Pinto-Bruno AC, Bighelli I, Berg-Weger M, van Straten A, Albanese E, Pot AM (2018) Online training and support programs designed to improve mental health and reduce burden among caregivers of people with dementia: A systematic review. J Am Med Dir Assoc 19, 200-206 e201.

[13] Morelli N, Barello S, Mayan M, Graffigna G (2019) Supporting family caregiver engagement in the care of old persons living in hard to reach communities: A scoping review. Health Soc Care Community 27, 1363-1374.

[14] Ruggiano N, Brown EL, Li J, Scaccianoce M (2018) Rural dementia caregivers and technology: What is the evidence? Res Gerontol Nurs 11, 216-224.

[15] Kovaleva M, Blevins L, Griffiths PC, Hepburn K (2019) An online program for caregivers of persons living with dementia: Lessons learned. J Appl Gerontol 38, 159-182.

[16] Beatty L, Binnion C (2016) A systematic review of predictors of, and reasons for, adherence to online psychological interventions. Int J Behav Med 23, 776-794.

[17] Preschl B, Maercker A, Wagner B (2011) The working alliance in a randomized controlled trial comparing online with face-to-face cognitive-behavioral therapy for depression. BMC Psychiatry 11, 189.

[18] Boot WR, Charness N, Czaja SJ, Sharit J, Rogers WA, Fisk AD, Mitzner T, Lee CC, Nair S (2015) Computer proficiency questionnaire: Assessing low and high computer proficient seniors. Gerontologist 55, 404-411.

[19] Harris PA, Taylor R, Thielke R, Payne J, Gonzalez N, Conde JG (2009) Research electronic data capture (REDCap)a metadata-driven methodology and workflow process for providing translational research informatics support. $J$ Biomed Inform 42, 377-381.

[20] Toye C, Lester L, Popescu A, McInerney F, Andrews S, Robinson AL (2014) Dementia Knowledge Assessment
Tool Version Two: Development of a tool to inform preparation for care planning and delivery in families and care staff. Dementia (London) 13, 248-256.

[21] Bedard M, Molloy DW, Squire L, Dubois S, Lever JA, O'Donnell M (2001) The Zarit Burden Interview: A new short version and screening version. Gerontologist 41, 652657.

[22] Al-Janabi H, Coast J, Flynn TN (2008) What do people value when they provide unpaid care for an older person? A meta-ethnography with interview follow-up. Soc Sci Med 67, 111-121.

[23] Reisberg B, Ferris SH, de Leon MJ, Crook T (1982) The Global Deterioration Scale for assessment of primary degenerative dementia. Am J Psychiatry 139, 1136-1139.

[24] Garcia-Alberca JM, Pablo Lara J, Gonzalez-Baron S, Barbancho MA, Porta D, Berthier M (2008) [Prevalence and comorbidity of neuropsychiatric symptoms in Alzheimer's disease]. Actas Esp Psiquiatr 36, 265-270.

[25] Serby M, Zucker D, Kaufman M, Franssen E, Duvvi K, Rypma B, Rotrosen J (1988) Clinical stages of dementia and the dexamethasone suppression test. Prog Neuropsychopharmacol Biol Psychiatry 12, 833-836.

[26] Kaufer DI, Cummings JL, Ketchel P, Smith V, MacMillan A, Shelley T, Lopez OL, DeKosky ST (2000) Validation of the NPI-Q, a brief clinical form of the Neuropsychiatric Inventory. J Neuropsychiatry Clin Neurosci 12, 233-239.

[27] Brodaty H, Donkin M (2009) Family caregivers of people with dementia. Dialogues Clin Neurosci 11, 217-228.

[28] Oliveira D, Zarit SH, Orrell M (2019) Health-promoting self-care in family caregivers of people with dementia: The views of multiple stakeholders. Gerontologist 59, e501e511.

[29] Wimo A, Reed CC, Dodel R, Belger M, Jones RW, Happich M, Argimon JM, Bruno G, Novick D, Vellas B, Haro JM (2013) The GERAS Study: A prospective observational study of costs and resource use in community dwellers with Alzheimer's disease in three European countries-study design and baseline findings. J Alzheimers Dis 36, 385-399.

[30] Reed C, Belger M, Dell'agnello G, Wimo A, Argimon JM, Bruno G, Dodel R, Haro JM, Jones RW, Vellas B (2014) Caregiver burden in Alzheimer's disease: Differential associations in adult-child and spousal caregivers in the GERAS Observational Study. Dement Geriatr Cogn Dis Extra 4, 51-64.

[31] NAC and AARP (2020) Caregiving in the U.S. 2020.

[32] Gilmore-Bykovskyi A, Johnson R, Walljasper L, Block L, Werner N (2018) Underreporting of gender and race/ethnicity differences in nih-funded dementia caregiver support interventions. Am J Alzheimers Dis Other Demen 33, 145-152.

[33] Pinquart M, Sorensen S (2011) Spouses, adult children, and children-in-law as caregivers of older adults: A metaanalytic comparison. Psychol Aging 26, 1-14.

[34] Napoles AM, Chadiha L, Eversley R, Moreno-John G (2010) Reviews: Developing culturally sensitive dementia caregiver interventions: Are we there yet? Am J Alzheimers Dis Other Demen 25, 389-406.

[35] Pleasant M, Molinari V, Dobbs D, Meng H, Hyer K (2020) Effectiveness of online dementia caregivers training programs: A systematic review. Geriatr Nurs 41, 921-935.

[36] Rosenthal R, Rubin DB (1979) A note on percent variance explained as a measure of the importance of effects. $J$ Appl Soc Psychol 9, 395-396. 